\title{
Training in Post Graduate Medical Education: Demands Rethinking
}

In general primary goal of doctor in care and well being of patients. This requires to acquire knowledge, learn clinical skills and develop professional attitudes. This can be achieved through a scheduled training curriculum identifying the central importance of competence-based learning, underpinned by structured, observational assessments. At the heart of the curriculum is patient safety and care within the framework of clinical governance. The emphasis on developing medical practitioners who are patient-focused and accountable to the public for delivering safe medical care is fundamental to creating the medical workforce of the future. It focuses on both clinical skills - with particular emphasis on chronic disease management and the care of acutely ill patients - as well as the development of more generic skills such as communications and team working. Doctors in training must develop the capacity to reflect on the relationship between personal performance and those outcomes and to learn from efforts to change

Bangladesh College of physicians and Surgeons (BCPS) as a pioneer and authorized organization in this field is playing pivotal role in health manpower development and maintenance of quality health services in the country. Despite its all-out effort, the optimization of the quality of its system and thus the outcome is sometimes questioned especially in the face of continuous piling of huge number of unsuccessful candidates in post graduate final examination. To overcome these, a number of relevant issues, related to training of doctors have been raised to address, viz. motivation of pursuit students, place of training, attitude \& opportunities of the trainers, monitoring of training and distribution \& number of trainees under a supervisor.

Lack of motivation of students is not straightway applicable to them as they entered the course through competitive examination conducted by the BCPS. Though controversies arises on inconsistent results in different sessions and in different specialties. Adequate and appropriate inputs from the senior teachers are earnestly sought; introduction of single best answer (SBA) type questions partially improved the assessment system but the target still remains to be reached. So, to develop a fair system of selection of optimum number of quality candidates for training is a crying need.

Institution and or place at which candidates take their clinical training have impact on their learning attitude and spectrum. It is the fact that all institutes where training is officially recognized are not equally equipped, both in infrastructures and patient management system; so the training outcome may vary.

Teachers, supervising postgraduate trainee are not always and equally oriented and optimized to training contents and modules due continuous updating. So regular refresher course, involving both senior and junior teachers supervising training might be of help.

In the existing system, a trainee, being placed to a training position at one or a number of recognized institutions is to complete the stipulated tenure to get a certificate duly signed both by the supervisor and hospital authority which confer him/her eligibility to seat for exam in clinical part. This mere certification of complete training is not ensuring the optimum learning as are reflected in their performance in exams. So stratification of learning in training period and structuring of each stratum with fixing up of time frame to complete each term through assessment is urgently warned.

We are already inundated with a number of qualified students, but do not have specific plan to train them. As the size of the enrolled students have outnumbered the minimum number of institutes and supervisors, we shall have to find out maximum number of students under a supervisors using all the training institutes of the country to train them within possible shortest period of time.

To address these issues, BCPS needs a coordinated approach involving the ministry of Health, Directorate 
of Health Services. NGOs and other health related private organization and institutes. Keeping in mind the existing and continuously increasing demand of specialist doctors, we shall have to have approximate calculation of number of candidates whom we can provide adequate training with possible drop out during and after completion of training. Accordingly, on standardizing the examination system, like the Royal Colleges of UK to select the top one third, expected number of candidates may be recruited from the top scorers not merely those who obtained qualified marks.

Total course of each specialty will have to be continuously updated by the qualified experience teacher of that specialty and learning level will have to be staged. Contents at each level will be structured. As candidates at various levels, need different level of training and all the recognized institutes are not equally equipped, institutions may be ranked according to their existing facilities and students as such may be placed in one of the hospitals of similar categories on rotation basis maintaining the hierarchy of training levels and institutions. Irrespective of experience, all the supervisors should have refresher course highlighting the update and implementation systems of training program from time and again.

Over all, we shall have to keep in mind that, postgraduate doctors we are producing are also teachers of our Medical institutes. So, component of postgraduate training should contain Continued Professional Development programme including Teaching methodology, Research and Assessment of performance.

Another important aspect and barrier of training is the payment, because a substantial proportion of our qualified students are not already in job. So this issue will have to be addressed appropriately.

The most important aspect of training is the monitoring and evaluation. Presently, a six monthly reporting by the supervisor is in practice. Further improvement may be done through regular evaluation and monitoring of training contents, quality of teaching and supervision, assessment and trainee progress. Both supervisors and trainee will have to contribute to the monitoring and evaluation process. Feedback from the supervisors will be systematically sought, analyzed and will be used as part of the monitoring process. Feedback from the trainee will also be sought confidentially regarding training, clinical experience and the quality of supervision. Comments, suggestions and advice may also be incorporated in the improvement of professional development process thus improved and modern treatment with time demanded health care services may be provided to the people of our community.

The training has no meaning or relevance if it is not set in the context of patient care. Its roots are in the service, not in the classroom. Our new doctors must be encouraged to immerse themselves in a clinical environment where this service is delivered.

(J Banagladesh Coll Phys Surg 2014; 32: 59-60)

\section{Prof. ABM Maksudul Alam \\ Councilor, BCPS \\ and Principal \& Professor of Anaesthesiology \\ Shaheed Suhrawardy Medical College \\ Sher-e-Bangla Nagar, Dhaka}

\section{References:}

1. A guide to CPSP training programs. Kerachi: Coll Physicians Surg Pak;2005

2. John SC Biggs: Postgraduate Medical Training in Pakistan: Observations and Recommendations; Journal of The College of Physicians and Surgeons of Pakistan 2006,Vol. (1):58-63

3. Curricullum for the foundation years in postgraguate education and training. By- The Foundation Programme Committee of Academyof Medical Royal Colleges, in co-operation with Modernising Medical Careers in the Departments 